\title{
Properties of Inertial Torques Acting on Gyroscopes
}

\author{
Ryspek Usubamatov* \\ Faculty of Transport and Manufacturing Engineering, Kyrgyz State Technical University, Kyrgyzstan
}

Submission: January 20, 2017; Published: March 28, 2018

*Corresponding author: Ryspek Usubamatov, Faculty of Transport and Manufacturing Engineering, Kyrgyz State Technical University named after I. Razzakov, Bishkek, Kyrgyzstan, Email: ryspek0701@yahoo.com

\begin{abstract}
The gyroscopic devices are primary arrangements for navigation and control systems that the contemporary aerospace, ships and other industries have been widely adopted. The main remarkable property of the gyroscope is represented in permanent maintaining the axis of a spinning rotor in a space. This gyroscope property is the result of action of the several internal inertial torques produced by the external load torque. Internal inertial torques of the gyroscope are generated by action of the components of centrifugal, common inertial and Coriolis forces as well as the change in the angular momentum. These inertial forces are produced by rotating mass elements and the center mass of the spinning rotor. The action of all gyroscope's internal torques are interrelated simultaneously around gyroscope axes, and are manifested the resistance and precession torques. The designers of gyroscopic devices can compute their acting forces and motions based on equations of the internal torques. However, gyroscope's inertial torques possess several properties, which correct usage enables getting correct results in computing of gyroscope parameters. This manuscript describes the properties of the inertial torques acting on a gyroscope that should be used for mathematical models of the gyroscope motions.
\end{abstract}

Keywords : Gyroscope theory; Torques; Motions; Forces

\section{Nomenclature}

$i$ - Index for $o x$ axis or oy

$J$ - Mass moment of inertia of a rotor's disc

$T_{a m, i}$ - Torque generated by change in angular momentum acting around axis $i$

$T_{c t i}, T_{c r, i}, T_{i n, i}$ - Torque generated by centrifugal, Coriolis and common inertial forces respectively and acting around axis $i$

\section{$T_{p, i}$ - Precession torque acting around axis $i$ \\ $T_{r, i}$ - Resistance torque acting around axis $i$ \\ $\omega$ - Angular velocity of a rotor \\ $\omega_{i}$ - Angular velocity of precession around axis $i$}

\section{Introduction}

From ancient time, the gyroscope was perplexing object because it moves in peculiar and intricate ways. Scientists become interested of its dynamics and famous mathematician L. Euler first laid the fundamental principle for the gyroscope theory in 1765. At those distant past the gyroscope effects were object of interest for brilliant scientists I. Newton, J-L. Lagrange, L. Poinsot, J.L.R. D'Alembert, P-S. Laplace, L. Foucaultand others that added new interpretations for gyroscope properties. The interesting and remarkable property of a gyroscope is its ability permanently maintain a reference direction in space that later was used in engineering. The profound interest to the gyroscope have emerged after industrial revolution, when gyroscope properties were investigated by researchers and published in scientific transactions [1-5]. The special gyroscope properties of a gyroscope make them extremely important in aerospace and ships industriesespecially for advanced navigation and control systems.

From the past century, innumerable publications and dozen theories were dedicated to applications of the gyroscope effects in engineering for mechanisms, devices and arrangements with the rotating parts [6,7]. Thegyroscope theory is the compulsory chapter in the fundamental textbooks of classical mechanics, particularly machine dynamics [8-10]. Nevertheless, the research transactions and textbooks do not fully explain the dynamics and physics of the gyroscope properties and its strange acting forces and motions still remain not adequately clear [11-12].

Contemporary high-tech engineering with the gyroscopic devices use for solutions of acting forces and motions the severe numerical models based on Lagrangian dynamics that is solved with computer's software [13]. This is forced solution. However, allknown mathematical models for gyroscope forces and motions contain assumptions and simplifications that cannot give correct results. The gyroscope effects and properties are described only by terms of the conservation of internal kinetic energy and by the action of the change in the 
angular momentum that is not enough for full explanation of properties [14-16]. Researchers intuitively pointed that the gyroscope effects and properties are the results of the action of the inertial forces, which are not represented by mathematical models. This is the reason that known mathematical models for the gyroscope properties do not match practical results $[17,18]$. Such situation with a gyroscope generated artificial terms as gyroscope resistance, gyroscope couple, gyroscope effects andothers, andeven seem to defy gravity and known physical laws. Unsolved gyroscope problems represent challenges for scientists that every year publish around hundred research results and discover new properties for gyroscopic devices $[19,20]$.

New investigations of the physical principles of gyroscope effects show that their nature is presented by the inertial forces of the mass elements of the spinning rotor. The gyroscope effects and properties are the results of acting centrifugal, common inertial and Coriolis forces as well as changes in the angular momentum of spinning rotor. All inertial forces generate the gyroscope's resistance and precession torques that are interrelated and occur at one time. The physics and mathematical models of these inertial torques are well described [21-23].

The gyroscopic devices can have different designs and can work at different conditions with several external loads. Computing the forces and motions for such conditions of gyroscope work is complex problem. This paper represents the analytical approach for the gyroscope motions around axes and describes properties of the inertial torques acting on a gyroscope. Themathematical model describes the action of the forces and motions accurately and validates by practical tests and furthermore gives explanation to the gyroscope properties.The mathematical model for the gyroscope motions under action of one load was tested on the Super Precision Gyroscope (Brightfusion Ltd, Abbeymead, UK).

\section{Methodology}

Recent analytical research into the physical principles of gyroscope effects have expressedthe new mathematical models forinertia forces acting in a gyroscope. These research results show that the origin of actual gyroscopic effects is more complex than presented in current publications. The action of the external load on a gyroscope around one axis that generates several internal resistance and precession torques acting around different axis. Equations of internal torques are shown in Table 1. Resistance torque is generated by the action of the centrifugal and Coriolis forces of the gyroscope's mass elements. The precession torque is generated by the action of the common inertial forces (for simplicity inertial forces) of the gyroscope's mass elements and by the well-known torque that is generated by the change in the angular momentum of the spinning rotor. These resistance and precession torques act simultaneously and interdependently, and are strictly perpendicular to each other around their axes [23].

Table 1: Equations of the gyroscope's internal torques.

\begin{tabular}{|c|c|}
\hline Type of torque generated by & Equation, (N.m) \\
\hline Centrifugalforces, $T_{c t, i}$ & \multirow{2}{*}{$T_{c t . i}=T_{i n . i}=2\left(\frac{\pi}{3}\right)^{2} J \omega \omega_{i}$} \\
\hline Common inertial forces, $T_{i n, i}$ & $T_{c r}=(8 / 9) J \omega \omega_{i}$ \\
\hline Coriolisforces, $T_{c r, i}$ & $T_{a m . i}=J \omega \omega_{i}$ \\
\hline $\begin{array}{c}\text { Change in angular momentum, } \\
T_{a m, i}\end{array}$ & \\
\hline
\end{tabular}

Table 1 contains the following symbols: $J$ is the rotor's massmoment of inertia around the spinning axle; $\omega_{i}$ is the angular velocity of precession of a spinning rotor around axis $i$ and $\omega$ is the angular velocity of a spinning rotor. The following analysis of the actions of several torques and motions around the two axes is used the system of subscripts signs. All components of the equations are marked by subscript signs that indicating the axis of action. For example, $T_{c r, x}$ is the Coriolis torque acting around axis $o x, \omega_{y}$ is the angular velocity of precession around axis oy, etc.

The external load torque acting on the gyroscope around one axis is generated the several internal torques acting around two axes. New mathematical models of the internal torques have discovered new gyroscopic properties and behavior of gyroscopic devices. All these inertial torques represent one system that is originated by the action of rotating mass-elements of the spinning rotor. Motions of the masselements in space generate inertial forces simultaneously acting in different directions at the same time. Action of any internal inertial force cannot be separated from the system of forces. Hence, several internal torques are manifested on the resistance and precession torques acting around two axes of a gyroscope. This is physical and fundamental principles of the gyroscope theory. The new equations for gyroscope internal torques enable formulating any mathematical models for the motions of the gyroscopic devices. This analytical approach allows the gyroscope properties and their physics to be described clearly by known laws of classical mechanics.

The formulated new equations for the resistance and precession torques that generated by one external torque being applied to the spinning rotor, show that these torques at defined proportion depend on the mass moment of inertia of a rotor and angular velocity of the spinning rotor, as well as on the angular velocity of its precession.

The torques generated by the centrifugal and inertial forces is represented by one equation. However, the action of these torques applied to different axis of the spinning rotor which perpendicular each other. The analytical expression of this equation describes two different properties of two 


\section{Robotics \& Automation Engineering Journal}

torques. This is unusual mathematical model and at first sight contradicts to rules of classical mechanics, but analytical development and physics of action of these torques is correct.

The external torque applied on a gyroscope produces the resistance torques generated by the centrifugal and Corioils forces. The torques generated by the inertial forces and by the rate change in the angular momentum are represented the precession torque. All torques represent one indivisible system that acting simultaneously and interdependently on a gyroscope. Separation of action of some torque from this system is impossible.

Equations in Table 1 represent the internal torques of the spinning rotor that are generated by the external or load torque $T$. The action of the external and internal torques in the gyroscope is represented the sequence chain of torques that activated according to the turn around axes. The load torque generates the internal torques and leads to manifestation of the gyroscope several properties that should be considered in sequence of action around axis. Figure 1 represents the spinning rotor that locates symmetrically relatively its supports and shows the load and internal torques acting in the gyroscope.

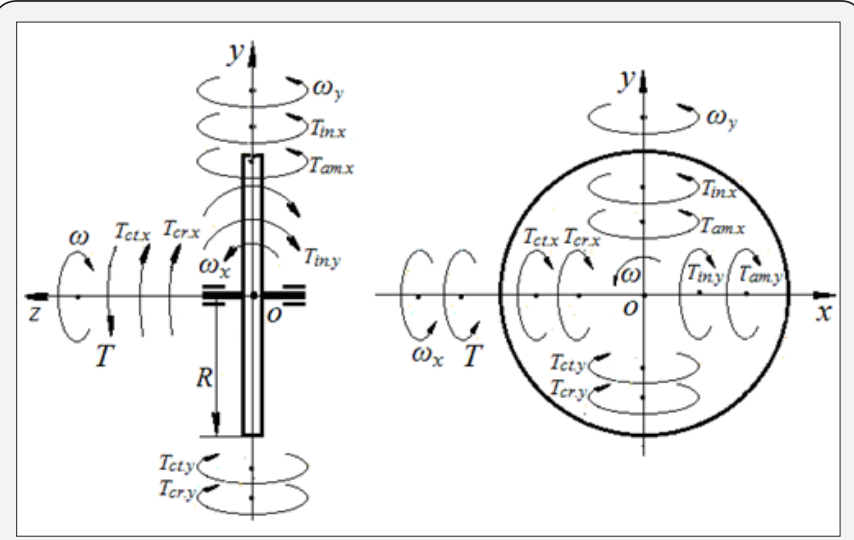

Figure 1: Load torque acting around axis ox and internal torques in the gyroscope.

The following steps of their manifestation consider the sequence action of the torques and gyroscope motions around axes:

\section{The gyroscope turns around axis $o x$}

- $\quad$ The load torque $T$ activates the resistance torques ( $T_{c t, x}$ and $T_{c r, x}$ ) that generated by the centrifugal and Coriolis forces, which are restraint torques that suppress the action of the load torque and acting around axis $o x$.

- Resistance torques ( $T_{c t, x}$ and $T_{c r, x}$ ) act opposite the action of the load torque $T$, but motion of the gyroscope at direction of the load torque.

- $\quad$ The load torque $T$ activates the precession torques ( $T_{i m, x}$ and $T_{a m, x}$ ) that generated by the inertial forces and the change of the angular momentum, which are load torques that are acting around axis oy. The resistance $\left(T_{c t, x}\right.$ and $T_{c r, x}$ ) and precession ( $T_{i n, x}$ and $T_{a m, x}$ ) torques are acting around axes $o x$ and oy respectively and simultaneously, and have one angular velocity of precession $\omega_{x}$. The action of these torques generated by one external torque $T$ that cannot be separated.

\section{The gyroscope turns around axis oy}

- The precession torques ( $T_{i n, x}$ and $\left.T_{a m, x}\right)$ that acting around axis oy generates the resistance torques ( $T_{c t, y}$ and $\left.T_{c r, y}\right)$ of centrifugal and Coriolis forces acting around axis oy .

- The precession ( $T_{i n, x}$ and $T_{a m, x}$ ) and resistance $\left(T_{c t, y}\right.$ and $T_{c r, y}$ ) torques acting around axis oy are generated the precession torques ( $T_{i n, y}$ and $T_{a n, y}$ ) of inertial forces and the change in the angular momentum

- Acting around axis $o x$. This precession torques $\left(T_{i n, y}\right.$ and $T_{a m, y}$ ) act opposite direction to the load torque $T$. The resistance torques ( $T_{c t, x}$ and $T_{c r, x}$ ) and the precession torques ( $T_{i n, y}$ and $T_{a m, y}$ ) in sum act opposite direction to the load torque $T$.

The action of the internal inertial torques in the gyroscope is the demonstration of interdependency and simultaneous action of its external and internal torques. Any change in values of internal torques originated at one axis lead to change in values of internal torques of other axis and vice versa. This statement expresses the equality of the kinetic energy of the spinning rotor which originated and distributed equally along the gyroscope axes $o x$ and oy. Internal torques, angular velocities of precessions and angular velocity of spinning rotor express the internal kinetic energy of the rotor that is constant for the given data of the gyroscope.

Table 2: Equations of the gyroscope's internal resistance and precession torques.

\begin{tabular}{|c|c|}
\hline Type of torque & Equation, (N.m) \\
\hline Resistance torque, $T_{c r, i}$ & {$\left[2\left(\frac{\pi}{3}\right)^{2}+\frac{8}{9}\right] J \omega \omega_{x}+\left[2\left(\frac{\pi}{3}\right)^{2}+1\right] J \omega \omega_{y}$} \\
\hline Precessiontorque, $T_{a m, i}$ & {$\left[2\left(\frac{\pi}{3}\right)^{2}+1\right] J \omega \omega_{x}-\left[2\left(\frac{\pi}{3}\right)^{2}+\frac{8}{9}\right] J \omega \omega_{y}$} \\
\hline
\end{tabular}

The action of the external torque on a gyroscope generates two resistances and two precession torques acting simultaneously and interdependently around two axes that strictly perpendicular to each other. This dual action of the external torque and interrelation of internal torques cannot be expressed and linked by mathematical equations. Represented properties and conditions of the gyroscope functioning demonstrate that all components of equations (Table 2) are interrelated each other and constant for the given gyroscope parameters. This property of the gyroscope does not contradict to principles of classical mechanics. 


\section{Robotics \& Automation Engineering Journal}

Represented above gyroscope's properties have defined explanations of their physics. Mathematical models of gyroscope torques and motions based on new analytical approaches are validated by practical tests. The internal torques that originated along each axis express the internal kinetic energy. The sum of these internal torques of one axis in absolute value is equal to the sum of the internal torques in absolute value of another axis. The actions of the internal torques originated along axes are not coincided with actions around axes. The resistance and precession torques are acting around two different axes. The equality of the internal kinetic energies of the spinning rotor two axes is represented by the equation of the action of the torques acting around axes ox and oy. This analytical expressionis represented by the following equation (Figure 1):

$$
-\left[2\left(\frac{\pi}{3}\right)^{2}+\frac{8}{9}\right] J \omega \omega_{x}-\left[2\left(\frac{\pi}{3}\right)^{2}+1\right] J \omega \omega_{y}=\left[2\left(\frac{\pi}{3}\right)^{2}+1\right] J \omega \omega_{x}-\left[2\left(\frac{\pi}{3}\right)^{2}+\frac{8}{9}\right] J \omega \omega_{y}
$$

where the sign $(-)$ and $(+)$ means the clockwise and counter clockwise directions of action the internal torques around two axes, respectively.

Simplification of Eq. (1) yields the following result:

$$
\omega_{y}=-\left(4 \pi^{2}+17\right) \omega_{x}
$$

Equation (2) demonstrates the actual ratio of the angular velocities of the gyroscope around two axes. Analysis of Eq. (2) shows the following peculiarity.Each axis contains resistance and precession torques only that originated on different axes, but these torques acting around two different axes and cannot be separated. Resistance torques of one axis are combined with precession torques of other axis, which actions can be contradicted. These combinations of internal inertial torques can change the magnitudes of the angular velocities around axes, but internal kinetic energies of each axis in absolute values remain constant in time according to the principle of conservation of mechanical energy. This statement enables the combination of the internal kinetic energies of the gyroscope around two axes to be described. The change in the value of external torque leads to change in value of internal kinetic energy of two axes. The values of internal torques are always less than the value of the permanent external torque that generates the internal kinetic energies around axes. Analysis and practical observation of the acting gyroscope with external load torque demonstrates the following properties:

a. For the permanent external torque, the internal kinetic energy of the spinning rotor is constant and depends on the angular velocity of the spinning rotor $\omega$ and angular velocity of precession $\omega_{x}$ around axis ox (Figure 1). The product of these two angular velocities always constant, $\omega \omega_{x}=$ const . Increasing or decreasing the angular velocity of the rotor leads to decreasing or increasing the angular velocity of precession respectively. b. The value of the internal torques and hence the angular velocity of precession $\omega x$ depends on the value of the external torque, on the rotor's mass moment of inertia and angular velocity of precession.

c. For the given value of the external torque and angular velocity of the rotor, there is limit of the angular velocity of precession $\omega x$.

d. Increasing the value of the external torque, while the angular velocity of the rotor is constant, leads to lose the ratio of the angular velocities of precession around two axes (Eq. 2).

e. The resulting internal torque acting around axis ox generates the resulting internal torque acting around axis oy that is manifested in the form of precession motions around axesthat values are significantly different.

f. Blocking of the gyroscope turn around any axis leads to elimination the angular velocities of precessions $\left(\omega_{x}=\right.$ 0 or $\omega_{y}=0$ ), i.e., all resistance and precession torques are deactivated.

g. Deactivation of the external torque $(\mathrm{T}=0)$ leads to deactivation of all resistance and the precession torques in spite of their acting on different axes.

h. The separation of the action of some internal inertial torque on the gyroscope is impossible.

i. The action of the load torque Taround axis oy generates the same sequence of action of the internal inertial torques as represented in Figure 1, but with different directions around axes oy and $o x$.

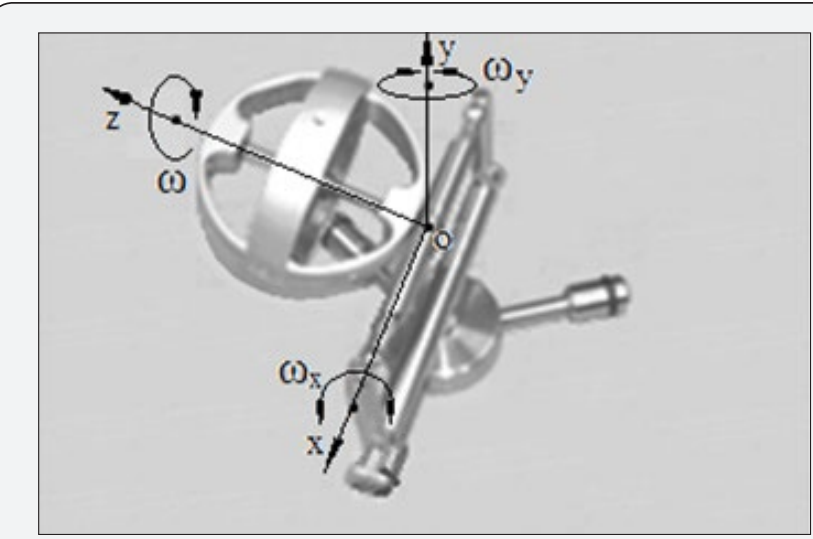

Figure 2 : The stand of the gyroscope with one side support.

Analysis of acting internal inertial torques on the gyroscope enables explaining the physics of these effects. At initial condition, the precession torque is generated by the several inertial forces and the change in the angular momentum of the spinning rotor acting around axis oy at condition when the gyroscope turns with the angular velocity of precession $\omega x$ around axis ox. Practically, the gyroscope precession motion 
around axes ox or oy can be blocked or braked by action of the friction forces. Figure 2 demonstrates the stand with a runninggyroscope of one side support. The stand assembled on the base of the Supper Precision Gyroscope "Brightfusion LTD" that enables applying two types load acting round two axes. The gyroscope with one side support assembled and fixed on the centre beam with the ability to freely rotate around axis ox on the journals of sliding bearings and that are disposed on the vertical arms of the frame. The framedesigned with two arms and the bar with vertical pivoted axle that disposed on the platform. The bar has the ability freely rotate around the fixed pivot (vertical axis ) on the platform.

This stand enables blocking and braking the motions of gyroscope around axis that demonstrating the following gyroscope properties.

\section{The blocking of the turn of the gyroscope around axis leads}

- To stop of rotation of the spinning rotor around axis i.e., the angular velocity and acceleration is eliminated, $\varepsilon_{y}=\omega \omega_{x}=0$, and hence, the angular velocity of the precession around axis ox is absent $\omega_{x}=0$. Then, the ratio of angular velocities around two axes (Eq. 2) is not maintained.

- $\quad$ The resistance torques originated and acted around axis $o x$ are deactivated. The precession torques originated around axis $o x$, but are acted around axis oy .

- $\quad$ Since the ratio of angular velocities around two axes (Eq. 2) is not maintained, a gyroscope turns free around axis $o x$ under action of the gravity force and generates the new internal inertial torques acting around axes $o x$ and $o y$ at new condition.

- As far as the gyroscope turn around axis oy is blocked, the resistance torques generated at condition of the free turn are deactivated around axisox, but the precession torques are acted around axis oy. This is unusual physical property of a gyroscope and manifestation of the interdependency of all internal torques acting around two axes.

\section{Braking the gyroscope rotation by action of the friction forces around axis oy leads}

- To proportional decreasing of the value of the precession torques, which is the load torques acting around axis oy.

- An action of the resistance torques around axis oy is result of the action the load precession torques. Hence, the value of this resistance torques that are involved in processes also have the same proportional decreasing.

- Decreasing of the value of the inertialtorques acting around axis leads to same decreasing of the value for the inertial torques around axis $o x$. This is result of the interdependency of the internal torques acting around two axes that reflects the principle of safety of the internal kinetic energy of the spinning rotor.

- Decreasing of the value of the inertial internal torques acting around axes and leads to increasing the action of the torque of the gyroscope weight. Hence, the angular velocities of gyroscope around axes $o x$ and $o y$ are increased.

The new analytical approach for the gyroscope internal inertial torques describes and represents in the new light the gyroscope properties. These properties of the gyroscope are manifested and validated by practical tests and observation. These particularities should be taken into account for engineering calculations of the gyroscope problems. New analytical approach to gyroscopic problems demonstrates that centrifugal, common inertial and Coriolis forces of the spinning rotor are active physical components as well as the change in the angular momentum. The latter one is one component among others and does not play the first role in the gyroscope physics. These four dynamical components act simultaneously and interdependently and actually represent fundamental principles and enable formulating the gyroscope theory. New mathematical models enable the describing all gyroscope properties and will be useful for modelling the behavior of the gyroscopic devices. The new analytical approach represents not only new method of analysis with respect to a gyroscope, but also new challenges for future studies of the gyroscopic devices.

\section{Results and Discussion}

The mathematical models for the gyroscope motions based on action of the resistance and precession torques that are generated by centrifugal, common inertial and Coriolis forces, as well as by the change in the angular momentum enable the description of all gyroscopic properties. New analytical approach to the gyroscope with symmetrical supports and one side support enables developing the equations for the gyroscope motions with two external load torques acting around two axes. One load represents the action of the gyroscope weight about horizontal axis and the other ones represents the action of the load torque around the vertical axis. The action of these torquesis combined with actions of the internal torques. All torques act simultaneously and interdependently in the gyroscope. The new analytical approach to gyroscopic problems demonstrates and explain the physical principles of acting torques on a gyroscope and its motions. The counter clockwise direction of the main load torque around vertical axis leads to the clockwise turn up of the gyroscope with one side support around horizontal axisox. This validation, that a gyroscope does not possess the anti-gravity effect. The clockwise direction of the load torque leads to the gyroscope 


\section{Robotics \& Automation Engineering Journal}

turn is opposite direction. The extra load torque of short time action leads to increasing the internal kinetic energy of inertial torques that manifest the oscillation (galloping) of the gyroscope. All mathematical models of the gyroscope motions with external loads are validated the gyroscope properties by practical observation.

\section{Conclusion}

The gyroscope theory in classical mechanics is one of the most complex and intricate theories in terms of analytical solutions. The known mathematical models of the gyroscope theory are mainly based on the actions of the external load torque and the change in the angular momentum of the spinning rotor. Numerical mathematical models for the gyroscope motions are complex and solving by computer's software that cannot explain the physics of acting forces. The known approaches leadtoo many assumptions and simplifications in the case of the unexplainable motions of the gyroscope devices. The nature of the gyroscope motions is more complex than presented in the known gyroscope theories. Practically, the centrifugal, common inertial and Coriolis forces and the change in the angular momentum of the spinning rotor are active physical components that describe all gyroscope properties. All these forces act in the gyroscope simultaneously and interdependently and represented the resistance and precession torques. The presentation of the action of theseinternal torques by analytical models of the gyroscope motions enables clear understandingthe physical process that results in the gyroscopic devices. The new analytical to the gyroscope problems is simple and can be used for engineering solutions of the gyroscopic devices.The new mathematical models of internal torques acting on a gyroscope represent fundamental principles for gyroscope theory that can solve all gyroscope problems and represent new challenges for future studies of gyroscopic devices.

\section{References}

1. Perry J (2012) Spinning Tops and Gyroscopic Motions, Literary Licensing, LLC, NV USA.

2. Cordeiro FJB (2015) The Gyroscope, Createspace, NV US.

3. Greenhill G (2015) Report on Gyroscopic Theory, Relnk Books, Fallbrook, USA.

4. Scarborough JB (2014) The Gyroscope Theory And Applications, Nabu Press, London, UK.

5. Neil B (2014) Gyroscope, The Charles Stark Draper Laboratory, Inc Cambridge, Massachusetts, UK.

6. Jonsson RM (2007) Gyroscope precession in special and general relativity from basic principles. American Journal of Physics 75: 463471.
7. Weinberg H (2011) Gyro Mechanical Performance: the most important parameter. Technical Article MS-2158, Analog Devices, Norwood, MA, p. 1-5.

8. Hibbeler RC, Yap KB (2013) Mechanics for Engineers - Statics and Dynamics, 13th ed. Prentice Hall, Pearson, Singapore.

9. Gregory DR (2006) Classical Mechanics, Cambridge University Press, New York, USA.

10. Aardema MD (2005) Analytical Dynamics. Theory and Application. Academic/Plenum Publishers, New York, USA.

11. Inampudi R, Gordeuk J (2016) Simulation of an Electromechanical Spin Motor System of a Control Moment Gyroscope. AIAA Guidance, Navigation, and Control Conference.

12. Liang WC, Lee SC (2013) Vorticity, gyroscopic precession, and spincurvature force. Physical Review D 87, 044024.

13. Klein F, Sommerfeld A (2008-2014) The theory of the top. Vol. I - IV. New York, NY: Springer, Birkhäuser, USA.

14. Zhang N, Ren YF, Li SK (2012) Research on Testing Method of Dynamic Characteristic for MEMS-Gyroscope. Advanced Materials Research, 346: 515-520.

15. Crassidis JL, Markley FL (2016) Three-Axis Attitude Estimation Using Rate-Integrating Gyroscopes. Journal of Guidance, Control, and Dynamics 39(7).

16. Gui H, Vukovich G, Xu S (2016) Attitude Stabilization of a Spacecraft with Two Parallel Control Moment Gyroscopes. Journal of Guidance, Control, and Dynamics 39(3): 728-735.

17. Stevenson D, Schaub H (2012) Nonlinear Control Analysis of a Double-Gimbal Variable Speed Control Moment Gyroscope. Journal of Guidance, Control, and Dynamics 35(3): 787-793.

18. Doupe C, Swenson ED (2016) Optimal Attitude Control of Agile Spacecraft Using Combined Reaction Wheel and Control Moment Gyroscope Arrays. (AIAA 2016-0675), AIAA Modeling and Simulation Technologies Conference.

19. Nanamori Y, Takahashi M (2015) An Integrated Steering Law Considering Biased Load sand Singularity for Control Moment Gyroscopes. (AIAA 2015-1091), AIAA Guidance, Navigation, and Control Conference.

20. Braun RD, Putnam ZR, Steinfeldt BA, Grant MJ, Barton G (2013) Advances in Inertial Guidance Technology for Aerospace Systems. (AIAA 2013-5123), AIAA Guidance, Navigation, and Control (GNC) Conference.

21. Usubamatov R (2014) Properties of Gyroscope Motion About One Axis. International Journal of Advancements in Mechanical and Aeronautical Engineering 2(1): 39-44.

22. Usubamatov R (2016) A Mathematical Model for Motions of Gyroscope Suspended from Flexible Cord. Cogent Engineering, Oktober.

23. Usubamatov R (2018) Inertial Forces Acting on Gyroscope. Journal of Mechanical Science and Technology 32(1): 101-108. 
This work is licensed under Creative

Commons Attribution 4.0 License

DOI: 10.19080/RAEJ.2018.02.555593
Your next submission with Juniper Publishers will reach you the below assets

- Quality Editorial service

- Swift Peer Review

- Reprints availability

- E-prints Service

- Manuscript Podcast for convenient understanding

- Global attainment for your research

- Manuscript accessibility in different formats

( Pdf, E-pub, Full Text, Audio)

- Unceasing customer service

Track the below URL for one-step submission https://juniperpublishers.com/online-submission.php 